Proc Indian Natn Sci Acad 81 No. 1 February 2015 Special Issue, pp. 86-94

(C) Printed in India.

DOI: $10.16943 /$ ptinsa/2015/v81i1/48055

\title{
QCD Thermodynamics at Finite Temperature and Finite Chemical Potential
}

\author{
NAJMUL HAQUE ${ }^{*}$ \\ Theory Division, Saha Institute of Nuclear Physics, 1/AF Bidhannagar, Kolkata 700064 India
}

(Received on 11 May 2014; Accepted on 9 September 2014)

\begin{abstract}
We calculate thermodynamic functions viz. pressure, change in pressure due to chemical potential, quark number density of hot and dense nuclear matter at finite temperature and chemical potential using threeloop Hard Thermal Loop perturbation theory.
\end{abstract}

Key Words : Quantum Chromodynamics; Quark Gluon Plasma; Hard Thermal Loop Perturbation Theory; Temperature; Chemical Potential

\section{Introduction}

Quantum chromodynamics (QCD) exhibits a rich phase structure and describes the propagation and interaction of quarks and gluons which are the fundamental constituents of all hadronic matter. Based solely on the QCD Lagrangian it is possible to calculate the QCD partition function at finite temperature and finite chemical potential and it results in the so-called equation of state (EoS). The determination of the QCD EoS and other thermodynamical quantities are extremely important to the phenomenology of the hot and dense nuclear matter commonly known as quark-gluon plasma (QGP). At this time, the most reliable method to calculate the QCD thermodynamic functions at finite temperature and zero (small) chemical potential is lattice gauge theory (Borsanyi et al., 2010, 2012a,b,c; Borsanyi 2013; Borsanyi et al., 2009, 2012, 2013; Bernard et al., 2005; Petreczky 2012). Importantly, lattice QCD can be used to probe the behaviour of QCD matter near the phase transition from the hadronic to the deconfined QGP phase. Near the phase transition temperature, the running coupling is large and non-perturbative methods like lattice QCD must be used. Finite temperature lattice QCD calculations are now quite stable; however, due to the sign problem, it is not straightforward to extend such calculations to finite baryon chemical potential. In practice, it is possible to obtain information about the behaviour of the thermodynamic functions at small baryon chemical potential

*Author for Correspondence : E-mail: najmul.haque@saha.ac.in 
by making a Taylor expansion of the partition function around zero baryonic chemical potential and extrapolating the result. This requires the calculation of various order quark-number susceptibilities evaluated at zero chemical potential. Since extrapolations based on a finite number of term, Taylor coefficients can only be trusted at small value of chemical potential and it would be nice to have an alternative framework for calculating the finite temperature and chemical potential QCD thermodynamic potential and associated quantities. This is important in light of the ongoing beam energy scan at the Relativistic Heavy Ion Collider (RHIC) in Brookhaven National Laboratory (BNL) and at Large Hadron Collider (LHC) in European Organization for Nuclear Research (CERN) and also at the forthcoming experiments at the Facility for Antiproton and Ion Research (FAIR) in Gesellschaft für Schwerionenforschung (GSI). As an alternative to lattice QCD calculations, one natural option is to compute the thermodynamic potential using perturbation theory at finite temperature and at finite chemical potential. In principle, this should work since, at sufficiently high temperature, the value of the strong coupling constant is small; however, one does not know a priori how large the temperature should be for this method to give result which are close to reality within a good approximation.

Unfortunately, it turns out that a strict expansion in the coupling constant converges only for temperatures many orders of magnitude higher than those relevant for heavy-ion collision experiments. The source of the poor convergence comes from contributions from soft momenta, $p \sim g T$. This suggests that one needs a way of reorganising the perturbative series which treats the soft sector more carefully. As a result, a gauge-invariant reorganising called hard-thermal-loop perturbation theory (HTLpt) was developed by Braaten and Pisarski in Braaten et al., (1990). HTL perturabtion has been used to calculate thermodynamic functions and other thermodynamical quantities at one loop (Andersen et al., 2000; Haque et al., 2010, 2011a,b; Chakraborty et al., 2002, 2003), at two loops (Andersen et al., 2002; Haque et al., 2013a,b) and at three loops at zero chemical potential (Su et al., 2010); (Andersen et al., 2009, 2010, 2011) as well as at finite chemical potential (Haque et al., 2014b,a) within HTL perturbation theory.

\section{Feynman Diagrams}

Feynman diagrams that will contribute to the QCD thermodynamics potential up to three-loop in HTL approximation are listed below: 

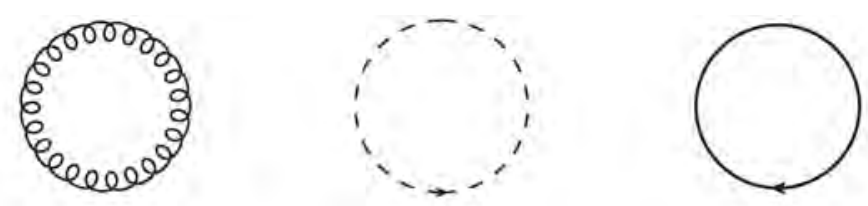

$\mathcal{F}_{1 k}^{\prime \prime}$

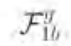

$\mathcal{F}_{1 h}^{\}}$

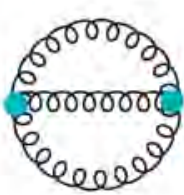

$\mathcal{F}_{3 a}^{\prime \prime}$

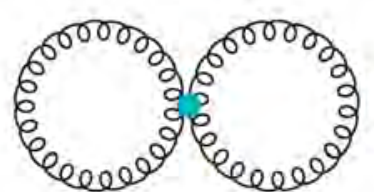

$\mathcal{F}_{2 b}^{\prime \prime}$
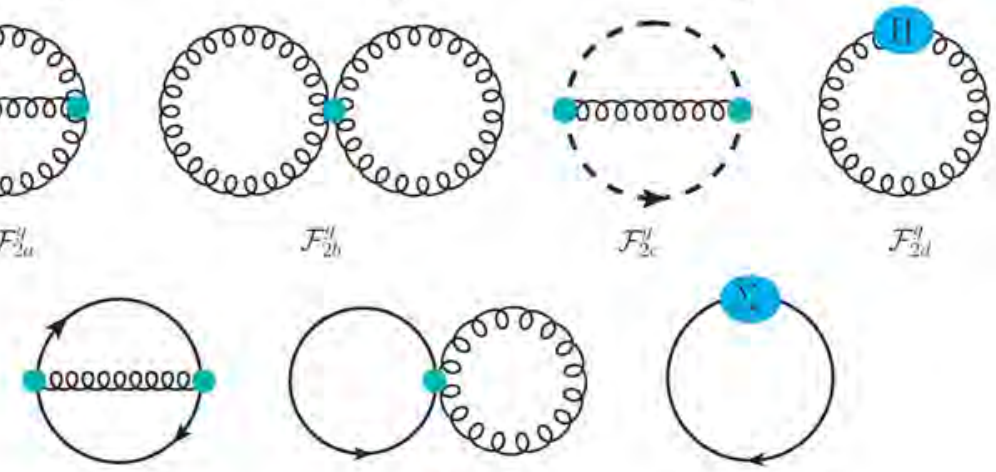

$\mathcal{F}_{2 o}$
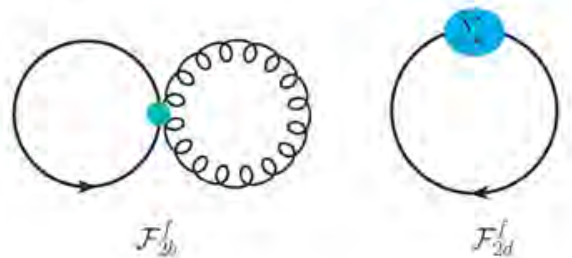

Fig. 1: One and two loop HTL Feynman diagrams contributing to the QCD thermodymanic potential

\section{Thermodynamics Potential}

The QCD thermodynamics potential up to three-loop HTL approximation including all the necessary counter term can be summerized as

$$
\begin{aligned}
\frac{\Omega_{\mathrm{NNLO}}}{\Omega_{0}} & =1+\frac{7}{4} \frac{d_{F}}{d_{A}}\left(1+\frac{120}{7} \hat{\mu}^{2}+\frac{240}{7} \hat{\mu}^{4}\right)-\frac{15}{4} \hat{m}_{D}^{3}+\frac{c_{A} \alpha_{s}}{3 \pi}\left[-\frac{15}{4}+\frac{45}{2} \hat{m}_{D}\right. \\
& \left.-\frac{135}{2} \hat{m}_{D}^{2}-\frac{495}{4}\left(\ln \frac{\hat{\Lambda}_{g}}{2}+\frac{5}{22}+\gamma_{E}\right) \hat{m}_{D}^{3}\right]+\left(\frac{c_{A} \alpha_{s}}{3 \pi}\right)^{2}\left[\frac{45}{4 \hat{m}_{D}}\right. \\
& -\frac{165}{8}\left(\ln \frac{\hat{\Lambda}_{g}}{2}-\frac{72}{11} \ln \hat{m}_{D}-\frac{84}{55}-\frac{6}{11} \gamma_{E}-\frac{74}{11} \frac{\zeta^{\prime}(-1)}{\zeta(-1)}+\frac{19}{11} \frac{\zeta^{\prime}(-3)}{\zeta(-3)}\right) \\
& \left.+\frac{1485}{4}\left(\ln \frac{\hat{\Lambda}_{g}}{2}-\frac{79}{44}+\gamma_{E}+\ln 2-\frac{\pi^{2}}{11}\right) \hat{m}_{D}\right]-\frac{s_{F} \alpha_{s}}{\pi}\left[\frac{5}{8}\left(1+12 \hat{\mu}^{2}\right)\left(5+12 \hat{\mu}^{2}\right)\right. \\
& \left.-\frac{15}{2}\left(1+12 \hat{\mu}^{2}\right) \hat{m}_{D}-\frac{15}{2}\left(2 \ln \frac{\hat{\Lambda}}{2}-1-\aleph(z)\right) \hat{m}_{D}^{3}+90 \hat{m}_{q}^{2} \hat{m}_{D}\right] \\
& +s_{2 F}\left(\frac{\alpha_{s}}{\pi}\right)^{2}\left[\frac { 1 5 } { 6 4 } \left\{35-32\left(1-12 \hat{\mu}^{2}\right) \frac{\zeta^{\prime}(-1)}{\zeta(-1)}+472 \hat{\mu}^{2}+1328 \hat{\mu}^{4}\right.\right. \\
& \left.+64\left(-36 i \hat{\mu} \aleph(2, z)+6\left(1+8 \hat{\mu}^{2}\right) \aleph(1, z)+3 i \hat{\mu}\left(1+4 \hat{\mu}^{2}\right) \aleph(0, z)\right)\right\}
\end{aligned}
$$




$$
\begin{aligned}
& \left.-\frac{45}{2} \hat{m}_{D}\left(1+12 \hat{\mu}^{2}\right)\right]+\left(\frac{s_{F} \alpha_{s}}{\pi}\right)^{2}\left[\frac{5}{4 \hat{m}_{D}}\left(1+12 \hat{\mu}^{2}\right)^{2}+30\left(1+12 \hat{\mu}^{2}\right) \frac{\hat{m}_{q}^{2}}{\hat{m}_{D}}\right. \\
& +\frac{25}{12}\left\{\left(1+\frac{72}{5} \hat{\mu}^{2}+\frac{144}{5} \hat{\mu}^{4}\right) \ln \frac{\hat{\Lambda}}{2}+\frac{1}{20}\left(1+168 \hat{\mu}^{2}+2064 \hat{\mu}^{4}\right)+\frac{3}{5}\left(1+12 \hat{\mu}^{2}\right)^{2} \gamma_{E}\right. \\
& -\frac{8}{5}\left(1+12 \hat{\mu}^{2}\right) \frac{\zeta^{\prime}(-1)}{\zeta(-1)}-\frac{34}{25} \frac{\zeta^{\prime}(-3)}{\zeta(-3)}-\frac{72}{5}\left[8 \aleph(3, z)+3 \aleph(3,2 z)-12 \hat{\mu}^{2} \aleph(1,2 z)\right. \\
& \left.\left.+12 i \hat{\mu}(\aleph(2, z)+\aleph(2,2 z))-i \hat{\mu}\left(1+12 \hat{\mu}^{2}\right) \aleph(0, z)-2\left(1+8 \hat{\mu}^{2}\right) \aleph(1, z)\right]\right\} \\
& \left.-\frac{15}{2}\left\{\left(1+12 \hat{\mu}^{2}\right)\left(2 \ln \frac{\hat{\Lambda}}{2}-1-\aleph(z)\right)\right\} \hat{m}_{D}\right] \\
& +\left(\frac{c_{A} \alpha_{s}}{3 \pi}\right)\left(\frac{s_{F} \alpha_{s}}{\pi}\right)\left[\frac{15}{2 \hat{m}_{D}}\left(1+12 \hat{\mu}^{2}\right)-\frac{235}{16}\left\{\left(1+\frac{792}{47} \hat{\mu}^{2}+\frac{1584}{47} \hat{\mu}^{4}\right) \ln \frac{\hat{\Lambda}}{2}\right.\right. \\
& -\frac{144}{47}\left(1+12 \hat{\mu}^{2}\right) \ln \hat{m}_{D}+\frac{319}{940}\left(1+\frac{2040}{319} \hat{\mu}^{2}+\frac{38640}{319} \hat{\mu}^{4}\right)-\frac{24 \gamma_{E}}{47}\left(1+12 \mu^{2}\right) \\
& -\frac{44}{47}\left(1+\frac{156}{11} \hat{\mu}^{2}\right) \frac{\zeta^{\prime}(-1)}{\zeta(-1)}-\frac{268}{235} \frac{\zeta^{\prime}(-3)}{\zeta(-3)}-\frac{72}{47}[4 i \hat{\mu} \aleph(0, z) \\
& \left.\left.+\left(5-92 \hat{\mu}^{2}\right) \aleph(1, z)+144 i \hat{\mu} \aleph(2, z)+52 \aleph(3, z)\right]\right\}+90 \frac{\hat{m}_{q}^{2}}{\hat{m}_{D}} \\
& +\frac{315}{4}\left\{\left(1+\frac{132}{7} \hat{\mu}^{2}\right) \ln \frac{\hat{\Lambda}}{2}+\frac{11}{7}\left(1+12 \hat{\mu}^{2}\right) \gamma_{E}+\frac{9}{14}\left(1+\frac{132}{9} \hat{\mu}^{2}\right)\right. \\
& \left.\left.+\frac{2}{7} \aleph(z)\right\} \hat{m}_{D}\right]
\end{aligned}
$$

where, with the standard normalization, the QCD Casimir numbers are $c_{A}=N_{c}, d_{A}=N_{c}^{2}-1, s_{f}=$ $N_{f} / 2, C_{F}=\left(N_{c}^{2}-1\right) / 2 N_{c}, s_{2 f}=C_{F} s_{f} . \Omega_{0}=-d_{A} \pi^{2} T^{4} / 45$, thermodynamic potential for free pureglue case. The sums over $f$ and $g$ include all quark flavors, $z_{f}=1 / 2-i \hat{\mu}_{f}$. The details about the functions $\aleph(z)$ and $\aleph(n, z)$ can be found in Haque et al $(2014 \mathrm{~b}, \mathrm{a})$. Here $m_{D}$ and $m_{q}$ are two parameters in HTL perturbation theory and we could identify these parameters as debye and thermal quark mass respectively. We have used here some scaled quantities as $\hat{m}_{D}=m_{D} / 2 \pi T, \hat{m}_{q}=m_{q} / 2 \pi T, \hat{\Lambda}=\Lambda / 2 \pi T$ and $\hat{\mu}=$ $\mu / 2 \pi T$. 

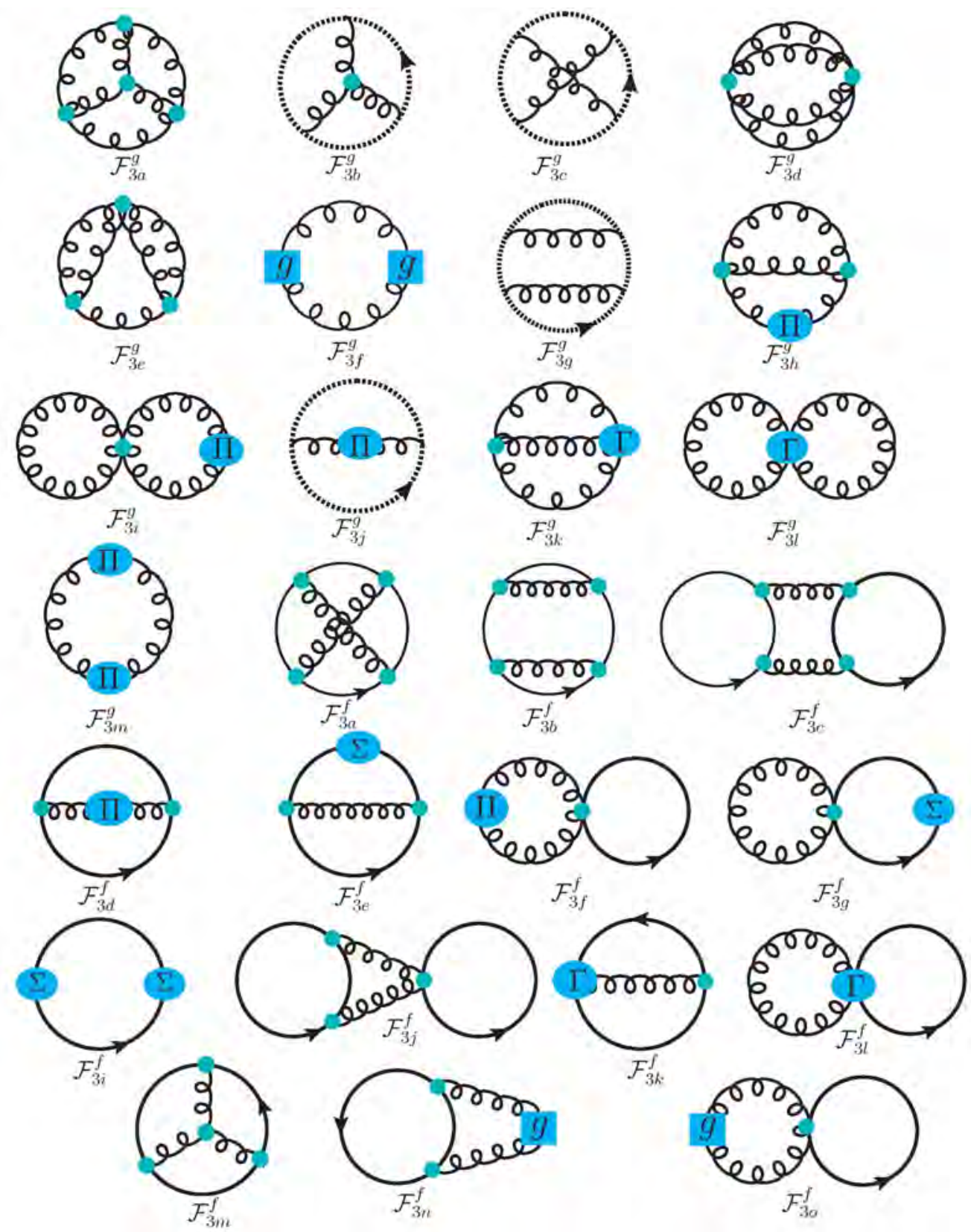

Fig. 2: Three loop HTL Feynman diagrams contributing to the QCD thermodymanic potential

\section{Pressure}

The QGP pressure can be obtained directly from the thermodynamic potential (2) as

$$
\mathcal{P}\left(T, \Lambda, \Lambda_{g}, \mu\right)=-\Omega_{\mathrm{NNLO}}\left(T, \Lambda, \Lambda_{g}, \mu\right),
$$


In Fig. 3 we compare the scaled NNLO HTLpt pressure for $\mu_{B}=0$ (A) and $\mu_{B}=400 \mathrm{MeV}$ (B) with available recent lattice data where $\mu_{B}=\sum_{N_{f}} \mu_{f}$. It is worth to mention here that for numerical results, we use
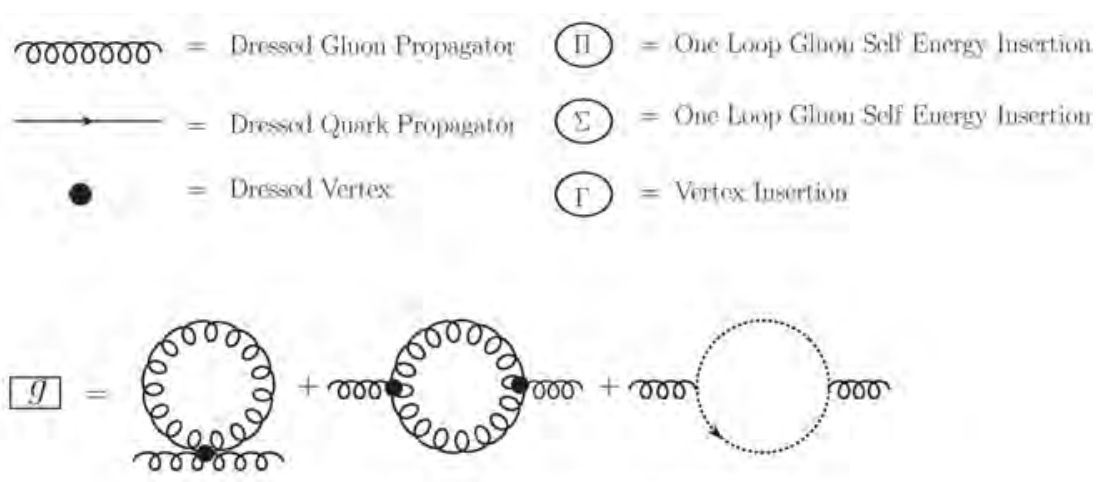

One-loop pue gauge pontribution to gluon self-energy
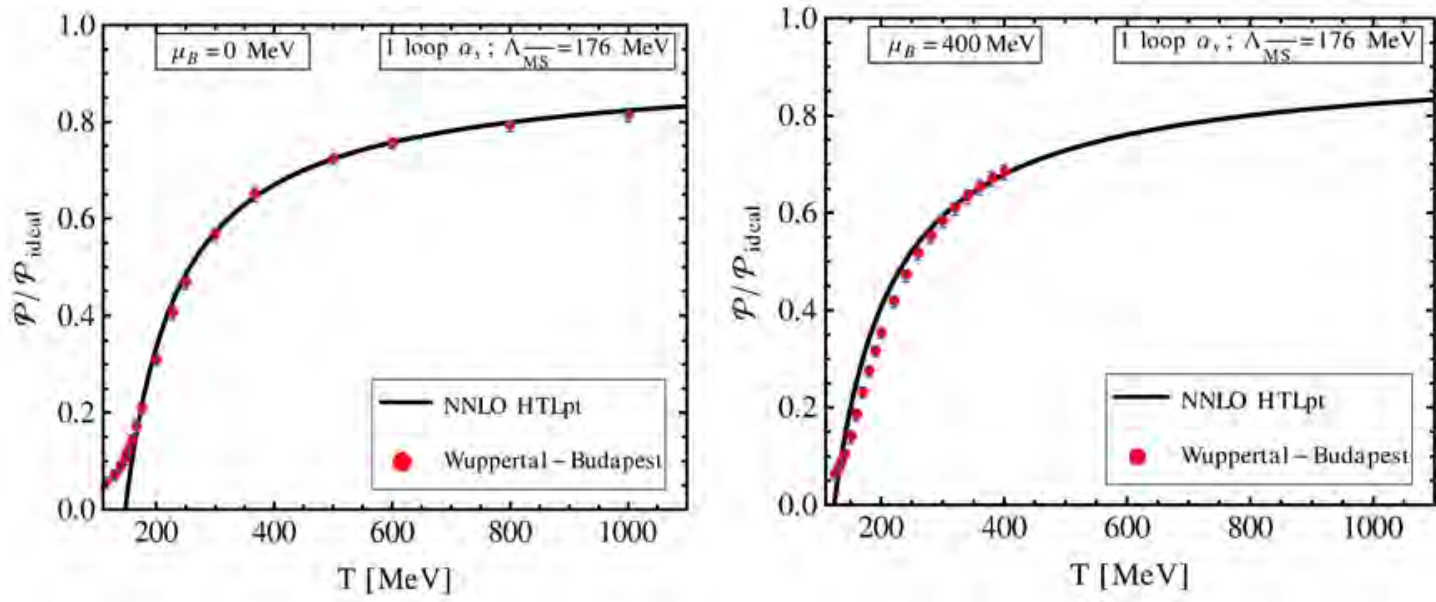

Fig. 3: Next-to-next leading order HTLpt QCD pressures at finite $\mu$ and zero $\mu$ scaled with free values for $\Lambda=2 \pi \sqrt{T^{2}+\mu^{2} / \pi^{2}}$ and $\Lambda_{g}=2 \pi T$ with lattice data from Borsanyi et al. (2012c) with one-loop running coupling constant

$$
\begin{aligned}
\hat{m}_{D}^{2}= & \frac{\alpha_{s}}{3 \pi}\left\{c_{A}+s_{F}\left(1+12 \hat{\mu}^{2}\right)+\frac{c_{A}^{2} \alpha_{s}}{12 \pi}\left(5+22 \gamma_{E}+22 \ln \frac{\hat{\Lambda}_{g}}{2}\right)\right. \\
& +\frac{c_{A} s_{F} \alpha_{s}}{12 \pi}\left(\left(9+132 \hat{\mu}^{2}\right)+22\left(1+12 \hat{\mu}^{2}\right) \gamma_{E}+2\left(7+132 \hat{\mu}^{2}\right) \ln \frac{\hat{\Lambda}}{2}+4 \aleph(z)\right) \\
& \left.+\frac{s_{F}^{2} \alpha_{s}}{3 \pi}\left(1+12 \hat{\mu}^{2}\right)\left(1-2 \ln \frac{\hat{\Lambda}}{2}+\aleph(z)\right)-\frac{3}{2} \frac{s_{2 F} \alpha_{s}}{\pi}\left(1+12 \hat{\mu}^{2}\right)\right\}, \\
\hat{m}_{q}^{2}= & \frac{C_{F} \alpha_{s}\left(1+4 \hat{\mu}^{2}\right)}{8 \pi} .
\end{aligned}
$$


We can also compute change in pressure due to finite chemical potential as

$$
\Delta \mathcal{P}(T, \Lambda, \mu)=\mathcal{P}(T, \Lambda, \mu)-\mathcal{P}(T, \Lambda, \mu=0)
$$

In the left panel of Fig. 4, we plot scaled $\Delta \mathcal{P}$ as a function $\mathrm{T}$ for different values of $\mu / T$.

\section{Number Density}

Number density is defined as the change of pressure w.r.t. chemical potential; $n(T, \Lambda, \mu)=\frac{\partial \mathcal{P}}{\partial \mu}$. In the right panel of Fig. 4 we plot scaled number density as a function $\mathrm{T}$ for different values of $\mu / T$.
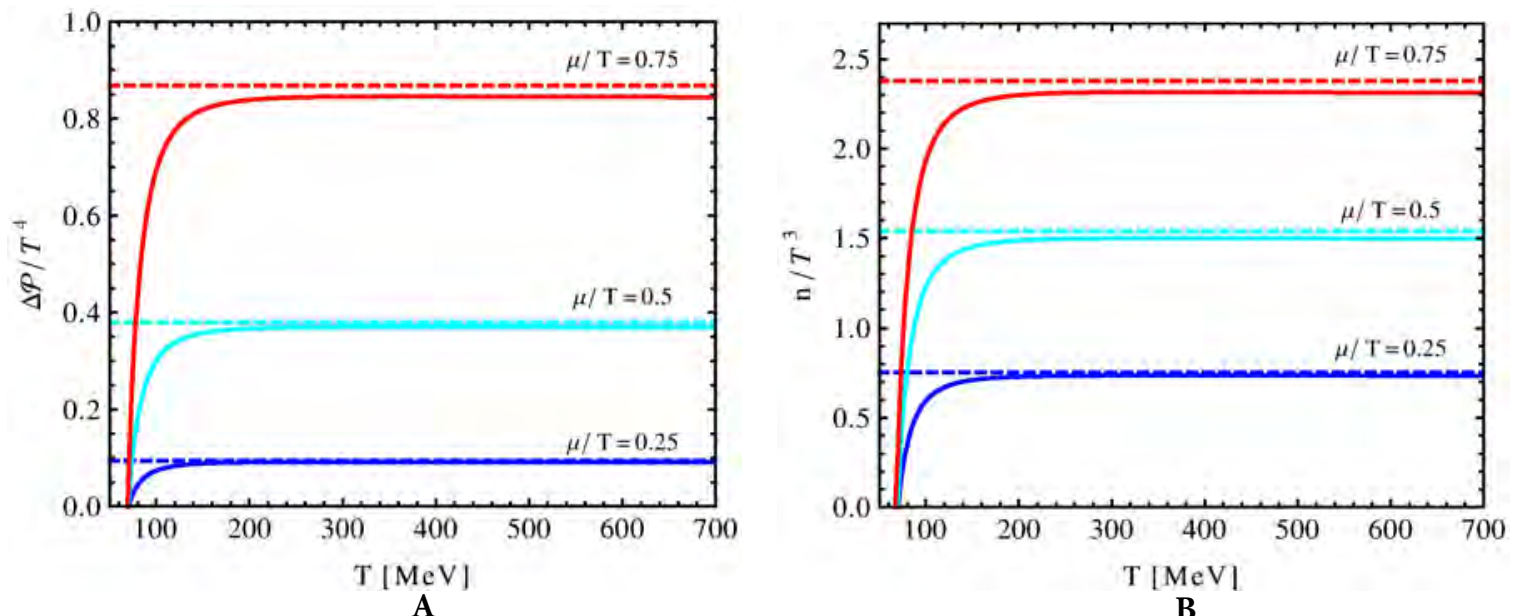

Fig. 4: A: Pressure difference due to finite $\mu$ as function of temperature for different values for $\mu / T$ with $\Lambda=2 \pi \sqrt{T^{2}+\mu^{2} / \pi^{2}}$. B: Quark number density for different values for $\mu / T$.

\section{Conclusion}

In this article, we have presented the results of a NNLO (three-loop) HTLpt calculation of the thermodynamic potential of QCD at finite temperature and chemical potential(s). Our final result is completely analytic, gauge invariant, and should be valid in the region of the phase diagram for which $\mu \lesssim 2 \pi T$. Based on the resulting thermodynamic potential we have calculated the pressure, change in pressure due to chemical potential and number density of the QGP. We have compared our result for pressure with recent lattice data and and we found very good agreement down to temperature as low as $200 \mathrm{Mev}$.

\section{References}

1. Andersen JO, Braaten E and Strickland M (2000) Hard thermal loop resummation of the free energy of a hot quark-gluon plasma, Phys Rev D61 074016 
2. Andersen JO, Petitgirard E and Strickland M (2002) Two loop HTL thermodynamics with quarks, Phys Rev D70 045001

3. Andersen JO, Strickland M and Su N (2009) Three-loop HTL Free Energy for QED, Phys Rev D80, 085015

4. Andersen JO, Strickland M and Su N (2010) Three-loop HTL gluon thermodynamics at intermediate coupling, JHEP 1008, 113

5. Andersen JO, Leganger LE, Strickland M and Su N (2011) Three-loop HTL QCD thermodynamics JHEP 1108 053

6. Bazavov A et al., (2009) Equation of state and QCD transition at finite temperature, Phys Rev D80 014504

7. Bazavov A et al., (HotQCD collaboration)(2012) Fluctuations and Correlations of net baryon number, electric charge, and strangeness: A comparison of lattice QCD results with the hadron resonance gas model, Phys Rev D86 034509

8. Bazavov A et al., (2013) Quark number susceptibilities at high temperatures, Phys Rev $\mathbf{8 8} 094021$

9. Bernard C et al., (MILC Collaboration) (2005) QCD thermodynamics with three flavors of improved staggered quarks, Phys Rev D71 034504

10. Borsanyi S et al., (2010) The QCD equation of state with dynamical quarks, JHEP 1011077

11. Borsanyi S et al., (2012a) Fluctuations of conserved charges at finite temperature from lattice QCD, JHEP 1201 138

12. Borsanyi S, Durr S, Fodor Z, Hoelbling C et al. (2012b) QCD thermodynamics with continuum extrapolated Wilson fermions I, JHEP 1208126

13. Borsanyi S et al., (2012c) QCD equation of state at nonzero chemical potential: continuum results with physical quark masses at order $\mu^{2}$, JHEP 08053

14. Borsanyi S (2013) Thermodynamics of the QCD transition from lattice, Nucl Phys A904-905 270c

15. Braaten E and Pisarski RD (1990) Soft Amplitudes in Hot Gauge Theories: A General Analysis, Nucl Phys B337 569

16. Chakraborty P, Mustafa MG and MH Thoma (2002) Quark number susceptibility in hard thermal loop approximation, Eur Phys J C23 591

17. Chakraborty P, Mustafa MG and MH Thoma (2003) Quark number susceptibility, thermodynamic sum rule and hard thermal loop approximation, Phys Rev $\mathbf{D 6 8} 085012$

18. Haque N et al., (2014a) Three-loop HTLpt Pressure and Susceptibilities at Finite Temperature and Density, Phys Rev D89 061701(R)

19. Haque $\mathrm{N}$ et al., (2014b) Three-loop HTLpt thermodynamics at finite temperature and chemical potential, JHEP 1405027

20. Haque N and Mustafa MG (2010) A Modified Hard Thermal Loop Perturbation Theory, arXiv:1007.2076.

21. Haque N and Mustafa MG (2011b) Quark Number Susceptibility and Thermodynamics in HTL approximation, Nucl Phys A862-863 271

22. Haque N, Mustafa MG and Strickland M (2013a) Two-loop HTL pressure at finite temperature and chemical potential, Phys Rev D87 105007 
23. Haque N, Mustafa MG and Strickland M (2013b) Quark Number Susceptibilities from Two-Loop Hard Thermal Loop Perturbation Theory, JHEP 1307184

24. Haque N, Mustafa MG and Thoma MH (2011a) Conserved Density Fluctuation and Temporal Correlation Function in HTL Perturbation Theory, Phys Rev D84 054009

25. Petreczky P (2012) Lattice QCD at non-zero temperature, J Phys G39 093002

26. Su N, Andersen JO and Strickland M (2010) Gluon Thermodynamics at Intermediate Coupling, Phys Rev Lett 104122003. 